\title{
Construcción de un diagrama de fases para la disolución ideal hexanos-ciclohexano, a partir de la determinación experimental de las presiones de vapor de cada
} sustancia

DOI: $10.46932 / \operatorname{sfjdv2n2-213~}$

Received in: March 1st, 2021

Accepted in: May 30th, 2021

\section{Fernanda Atondo Hernández}

Estudiante de noveno semestre de Ingeniería Química, Universidad Anáhuac México Norte. Universidad Anáhuac México Norte: Av. Universidad Anáhuac 46, Col. Lomas Anáhuac, Huixquilucan, Estado de México. C.P. 52786.

Correos: feratondo98@gmail.com; fernanda.atondohe@anahuac.mx

\section{Juan Daniel López Melo}

Estudiante de noveno semestre de Ingeniería Química, Universidad Anáhuac México Norte. Universidad Anáhuac México Norte: Av. Universidad Anáhuac 46, Col. Lomas Anáhuac, Huixquilucan, Estado de México. C.P. 52786.

Correos: juandaniel.lopez05@gmail.com ; juan.melo@anahuac.mx

\section{Ximena Villegas Pañeda}

Doctora en Ingeniería Química, profesora de la Universidad Anáhuac México Norte. Universidad Anáhuac México Norte: Av. Universidad Anáhuac 46, Col. Lomas Anáhuac, Huixquilucan, Estado de México. C.P. 52786.

Correo: ximena.villegas@anahuac.mx

\author{
Alma Delia Rojas Rodríguez \\ Maestra en Ingeniería Química, profesora de la Universidad Anáhuac México Norte. \\ Universidad Anáhuac México Norte: Av. Universidad Anáhuac 46, Col. Lomas Anáhuac, Huixquilucan, \\ Estado de México. C.P. 52786. \\ Correo: alma.rojas@anahuac.mx
}

\section{RESUMEN}

Los diagramas de fases son herramientas que sirven para predecir las fases presentes de cada componente en un sistema termodinámico, permiten conocer las condiciones de temperatura, presión y composición a las que se encuentra una sustancia, conocer las solubilidades de un elemento o compuesto en otro y poder especificar las variables necesarias en un proceso para determinar las fases del sistema cuando está en equilibrio. Son la herramienta principal de un ingeniero químico para poder entender y predecir varios aspectos de los materiales.

\section{INTRODUCCIÓN}

Se construyó un diagrama de fases para la disolución ideal de hexanos-ciclohexano (usando los cinco isómeros del hexano). El diagrama de fases binario de esta disolución ideal no había sido reportada anteriormente y se logró obtener dicho diagrama por medio del uso de hojas de cálculo, brindando mayor facilidad a los estudiantes que no cuentan con simuladores o programas para la obtención de diagramas de fases. 


\section{OBJETIVO}

- Determinar los valores experimentales de temperatura de una disolución ideal de hexanosciclohexano a diferentes composiciones y calcular las presiones de vapor, mediante la ecuación de Antoine, para construir un diagrama de fases binario.

\section{MARCO TEÓRICO}

Los diagramas de fases son representaciones gráficas de los componentes y las fases que se encuentran presentes en un sistema termodinámico a diferentes condiciones de presión, temperatura y composición. Los diagramas de fases, se construyen basándose en las condiciones de equilibrio, permiten determinar si la fase es termodinámicamente estable o no.

La ley de Dalton establece que en una mezcla de gases, cada gas ejerce una presión como si los otros gases no estuvieran presentes. La presión específica de un gas determinado en un mezcla se llama presión parcial (pi). La presión total se calcula sumando las presiones parciales de todos los gases que la componen.

Una disolución ideal es aquella en la que las moléculas de las especies son tan semejantes unas a otras, que las moléculas de uno de los componentes pueden sustituir a las del otro sin variar la estructura espacial de la disolución o de la energía de las interacciones intermoleculares. Una disolución ideal sigue la Ley de Raoult en todo el intervalo de concentraciones. La ley de Raoult permite calcular la presión de vapor de una sustancia cuando está formando parte de una disolución ideal, conociendo su presión de vapor cuando está pura (a la misma temperatura) y la composición de la disolución ideal en términos de fracción molar.

$$
P_{1}=x_{1} P_{1}^{\underline{o}}
$$

$P_{1}=$ presión de vapor del solvente, habiendo agregado el soluto

$x_{1}=$ fracción mol del componente de la solución

$P_{1}^{o}=$ presión de vapor del solvente puro

\section{METODOLOGÍA}

Se seleccionaron las sustancias hexanos(1) y ciclohexano(2) para construir el diagrama, debido a que forman una disolución ideal en todas sus composiciones. 
1. Se prepararon las disoluciones ideales de ciclohexano y hexanos en 6 tubos de ensayo de la siguiente manera:
1 a) $5 \mathrm{~mL}$ de hexanosy $0 \mathrm{~mL}$ de ciclohexano

$1 \mathrm{f}) 0 \mathrm{~mL}$ de hexanosy de precipitados de 150 $\mathrm{mL}$ aceite de cocina

3. En el tubo de ensaye de cada disolución, se introdujo un tubo capilar cerrado
4. Se colocó el tubo de ensaye (con la muestra y el capilar) dentro del vaso de precipitados con aceite en una parrilla previamente calentada.
9. Se hicieron los cálculos necesarios para la obtención del diagrama de fases temperatura vs composición
1 b) $4 \mathrm{~mL}$ de hexanos y $1 \mathrm{~mL}$ de ciclohexano

1 e) $1 \mathrm{~mL}$ de hexanosy $4 \mathrm{~mL}$ de ciclohexano 1c) $3 \mathrm{~mL}$ de hexanosy $2 \mathrm{~mL}$ de ciclohexano

1 d) $2 \mathrm{~mL}$ de hexanosy $3 \mathrm{~mL}$ de ciclohexano

\section{Se midió la} temperatura, colocando un termopar dentro del tubo de ensaye con la muestra y el capilar.
6. En el momento en el que se ve el burbujeo (ebullición), en forma de un rosario, porque el burbujeo se da repetidas veces, se registró la temperatura de ebullición.

$$
\text { ada. }
$$

\section{RESULTADOS}

Se prepararon las mezclas ya descritas en la metodología, obteniendo los siguientes datos de temperaturas de ebullición:

Para obtener los porcentajes de acuerdo con los mililitros:

$5 \mathrm{~mL}-100 \%$

$4 \mathrm{~mL}--80 \%$

\section{Todas las muestras,} al finalizar el experimento se vaciaron en un vaso de precipitados, para ser tratados posteriormente.

\section{Se repitió el} procedimiento con las muestras de la a) a la f), registrando las temperaturas de ebullición y las composiciones de cada muestra. 
Tabla 1. Resultados obtenidos en el laboratorio

\begin{tabular}{|c|c|c|}
\hline \multicolumn{2}{|c|}{ Composición molar fase líquida $(\mathrm{x})$} & \\
\hline Hexanos $(1)$ & Ciclohexano(2) & $\begin{array}{c}\text { Temperatura de ebullición } \\
\left({ }^{\circ} \mathrm{C}\right)\end{array}$ \\
\hline 1.0 & 0.0 & 54 \\
\hline 0.8 & 0.2 & 56 \\
\hline 0.6 & 0.4 & 63 \\
\hline 0.4 & 0.6 & 67 \\
\hline 0.2 & 0.8 & 71 \\
\hline 0.0 & 1.0 & 59 \\
\hline
\end{tabular}

Se consideró la presión total fue la presión de la Ciudad de México:

$\mathrm{PT}=586 \mathrm{mmHg}$

Al haber empleado la sustancia de Hexanos, se calcularon las presiones de saturación de todos los isómeros del hexano utilizando la ecuación de Antoine, y se sacó un promedio de ellas a diferentes temperaturas.

En las siguientes tablas se muestran los resultados obtenidos. Teniendo las siguientes constantes para los isómeros del hexano y el ciclohexano:

Tabla 2. Constantes de Antoine para las presiones de saturación de los isómeros del hexano

\begin{tabular}{|c|c|c|c|}
\hline Sustancia & A & B & C \\
\hline Hexano & 6.87024 & 1168.72 & 224.21 \\
\hline 2-metilpentano & 6.8391 & 1135.41 & 226.572 \\
\hline 3-metilpentano & 6.81887 & 1152.368 & 227.129 \\
\hline 2,2-dimetilbutano & 6.75483 & 1081.176 & 229.343 \\
\hline 2,3-dimetilbutano & 6.80983 & 1127.187 & 228.9 \\
\hline
\end{tabular}

Tabla 2.1 Presiones de vapor obtenidas mediante la ecuación de Antoine para cada sustancia a la temperatura obtenida experimentalmente de $54^{\circ} \mathrm{C}$ y su promedio:

\begin{tabular}{|c|c|}
\hline Sustancia & Presión $(\mathrm{mmHg})$ \\
\hline Hexano & 467.07 \\
\hline 2-metilpentano & 619.91 \\
\hline 3-metilpentano & 524.56 \\
\hline 2,2-dimetilbutano & 869.05 \\
\hline
\end{tabular}




\begin{tabular}{|c|c|} 
2,3-dimetilbutano & 669.00 \\
\hline Promedio: & 629.92 \\
\hline
\end{tabular}

Tabla 2.2 Presiones de vapor obtenidas mediante la ecuación de Antoine para cada sustancia a la temperatura obtenida experimentalmente de $56^{\circ} \mathrm{C}$ y su promedio:

\begin{tabular}{|c|c|}
\hline Sustancia & Presión $(\mathrm{mmHg})$ \\
\hline Hexano & 500.45 \\
\hline 2-metilpentano & 662.17 \\
\hline 3-metilpentano & 560.72 \\
\hline 2,2-dimetilbutano & 924.25 \\
\hline 2,3-dimetilbutano & 713.50 \\
\hline Promedio: & 672.22 \\
\hline
\end{tabular}

Tabla 2.3 Presiones de vapor obtenidas mediante la ecuación de Antoine para cada sustancia a la temperatura obtenida experimentalmente de $59^{\circ} \mathrm{C}$ y su promedio:

\begin{tabular}{|c|c|}
\hline Sustancia & Presión $(\mathrm{mmHg})$ \\
\hline Hexano & 554.05 \\
\hline 2-metilpentano & 729.76 \\
\hline 3-metilpentano & 618.62 \\
\hline 2,2-dimetilbutano & 1012.07 \\
\hline 2,3-dimetilbutano & 784.56 \\
\hline Promedio: & 739.81 \\
\hline
\end{tabular}

Tabla 2.4 Presiones de vapor obtenidas mediante la ecuación de Antoine para cada sustancia a la temperatura obtenida experimentalmente de $63^{\circ} \mathrm{C}$ y su promedio:

\begin{tabular}{|c|c|}
\hline Sustancia & Presión (mmHg) \\
\hline Hexano & 632.44 \\
\hline 2-metilpentano & 828.14 \\
\hline 3-metilpentano & 702.99 \\
\hline 2,2-dimetilbutano & 1138.98 \\
\hline 2,3-dimetilbutano & 887.72 \\
\hline Promedio: & 838.05 \\
\hline
\end{tabular}


Tabla 2.5 Presiones de vapor obtenidas mediante la ecuación de Antoine para cada sustancia a la temperatura obtenida experimentalmente de $67^{\circ} \mathrm{C}$ y su promedio:

\begin{tabular}{|c|c|}
\hline Sustancia & Presión $(\mathrm{mmHg})$ \\
\hline Hexano & 719.31 \\
\hline 2-metilpentano & 936.54 \\
\hline 3-metilpentano & 796.10 \\
\hline 2,2-dimetilbutano & 1277.72 \\
\hline 2,3-dimetilbutano & 1001.10 \\
\hline Promedio: & 946.15 \\
\hline
\end{tabular}

Tabla 2.6 Presiones de vapor obtenidas mediante la ecuación de Antoine para cada sustancia a la temperatura obtenida experimentalmente de $71^{\circ} \mathrm{C}$ y su promedio

\begin{tabular}{|c|c|}
\hline Sustancia & Presión $(\mathrm{mmHg})$ \\
\hline Hexano & 815.25 \\
\hline 2-metilpentano & 1055.64 \\
\hline 3-metilpentano & 898.53 \\
\hline 2,2-dimetilbutano & 1428.97 \\
\hline 2,3-dimetilbutano & 1125.34 \\
\hline Promedio: & 1064.75 \\
\hline
\end{tabular}

A partir de las temperaturas de ebullición obtenidas experimentalmente, las composiciones del líquido establecidas y empleando la presión parcial de cada muestra como el promedio de las presiones parciales de cada isómero del hexano, se calcularon las composiciones en el vapor con los siguientes datos: $\mathrm{P}_{1}=$ el promedio de las presiones de saturación a $54^{\circ} \mathrm{C}=629.92 \mathrm{mmHg}$. De igual manera se realizó para cada composición de vapor, usando las temperaturas y composiciones del líquido, como se muestra en la siguiente tabla:

Tabla 2.7 Temperaturas y composiciones en el líquido $\left(\mathrm{x}_{1}\right) \mathrm{y}$ el vapor $\left(\mathrm{y}_{1}\right)$ calculadas a partir del promedio de hexanos para la obtención del diagrama de fases

\begin{tabular}{|c|c|c|}
\hline $\mathrm{T}\left({ }^{\circ} \mathrm{C}\right)$ & $\mathrm{x}_{1}$ & $\mathrm{y}_{1}$ \\
\hline 54 & 1 & 1 \\
\hline 56 & 0.8 & 0.92 \\
\hline 59 & 0.6 & 0.76 \\
\hline 63 & 0.4 & 0.57 \\
\hline 67 & 0.2 & 0.32 \\
\hline
\end{tabular}




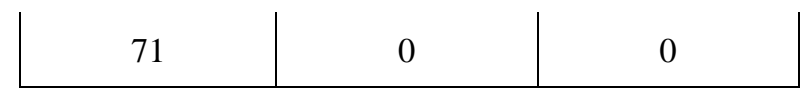

Con estos datos, se graficaron las curvas del líquido y del vapor contra la temperatura en grados Celsius, es decir, que el diagrama de fases queda en función de los hexanos:

Figura 1. Diagrama de fases hexanos-cilcohexano

\section{Temperatura-Composición}

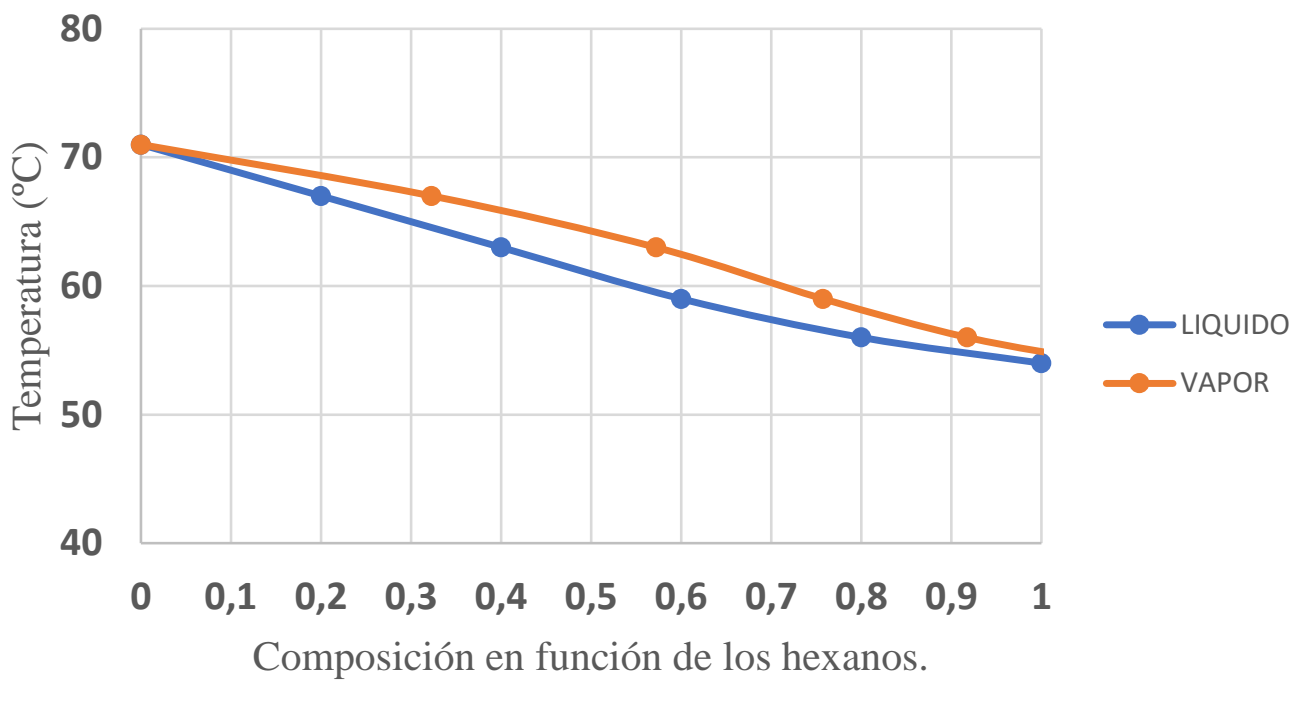

\section{ANÁLISIS DE RESULTADOS}

Dado que se usó la sustancia hexanos, para elaborar el diagrama de fases, y esa sustancia contiene cinco isómeros del hexano, se tuvo que hacer el ajuste para calcular las presiones de saturación con cada temperatura obtenida experimentalmente para cada uno de los cinco isómeros, con sus respectivas constantes de Antoine y al final se obtuvo un promedio de esas presiones. La importancia de hacer el promedio de las presiones de saturación de los isómeros del hexano, radica en que la presión atmosférica de la ciudad de México es de $586 \mathrm{mmHg}$ y las sustancias se evaporan a distintas temperaturas, pues no están a las condiciones normales de presión y temperatura, y conociendo que el punto de ebullición normal del hexano era $68^{\circ} \mathrm{C}$ y del ciclohexano $81^{\circ} \mathrm{C}$, en las lecturas de la temperatura de ebullición se registraron valores entre los $54^{\circ} \mathrm{C}$, esto por haber empleado la mezcla de hexanos y de $71^{\circ} \mathrm{C}$ en el ciclohexano, por las condiciones distintas de presión y temperatura de la ciudad de México. A partir de lo anterior, se hizo el cálculo de la composición de vapor de la disolución ideal, la cual obedece a la Ley de Raoult en todo intervalo de composiciones, con estos datos fue posible construir el diagrama de fases, como se muestra en la figura 1. 


\section{CONCLUSIONES}

La relevancia del proyecto radica en que se logró obtener el diagrama de fases de dos sustancias de manera experimental, sino que, se logró obtener un diagrama de fases de una sustancia, junto con otra que contenía los cinco isómeros del hexano, por medio de las presiones de vapor de cada uno de los isómeros del hexano, pudiendo concluir, que el hexano con sus isómeros, y el ciclohexano, se logran comportar como una disolución ideal. Es relevante también, porque se logró obtener el diagrama por medio del uso de hojas de cálculo, lo cual brinda mayor facilidad a los estudiantes que no cuentan con simuladores o programas para la obtención de diagramas de fases.

En este proyecto se escogió una disolución binaria ideal no usada anteriormente para construir un diagrama de fases binario a las condiciones de presión constante de la Ciudad de México (586 mmHg).

\section{AGRADECIMIENTOS}

Agradecemos al Doctor Víctor Hugo del Valle Muñoz, por permitirnos el uso del laboratorio de Química Orgánica para el desarrollo de este proyecto, así como a la Dirección de Investigación de la Universidad Anáhuac México por el patrocinio para participar en el $54^{\circ}$ Congreso Mexicano de Química, $38^{\circ}$ Congreso Nacional de Educación Química y Expoquímica 2019 de la Sociedad Química de México. 


\section{REFERENCIAS}

- $\quad$ Atkins, P. W., Fisicoquímica, $3^{\text {a }}$ Edición, USA, Addison-Wesley Iberoamericana, 1991.

- $\quad$ Atondo, F. y López, J. (2019, octubre) Construcción de un diagrama de fases para la disolución ideal hexanos-ciclohexano, a partir de la determinación experimental de las presiones de vapor de cada sustancia. Comunicación presentada en el $54^{\circ}$ Congreso Mexicano de Química, $38^{\circ}$ Congreso Nacional de Educación Química y Expoquímica, Puebla, México.

- $\quad$ Castellan, G. W., Fisicoquímica, 2a Edición, USA, Addison-Wesley Iberoamericana, 1987.

- Chang, R. (2002). QUÍMICA, séptima edición. Colombia: McGraw-Hill Companies, Inc.

- $\quad$ Hillert, M., Phase Equilibria, Phase Diagrams and Phase Transformations : Their Thermodynamic Basis, $1^{a}$ Edición, Reino Unido, Cambridge University Press, 1998

- Jaramillo, O. (2007) Ley de Raoult. Recuperado el: 10/05/2019. Disponible en: http://www.cie.unam.mx/ ojs/pub/Liquid3/node22.html

- Smith, Van Ness, Abbott (1997) Introducción a la termodinámica en ingeniería química. (5a edición). México: McGraw Hill.

- Universidad de las Islas Baleares (2011) Disoluciones ideales. Recuperado el: 11/05/2019. Disponible en: http://facultatciencies.uib.cat/prof/juan.frau/qfI/teoria/tema11.pdf

- Universidad Politécnica de Cartagena. Estados de la materia. Disoluciones. Recuperado el: 28/06/2021. Disponible en el sitio web: https://ocw.bib.upct.es/pluginfile.php/9626/mod_resource/content/2/Tema_4_Estados_de_agregacion_y _Disoluciones.pdf 